\title{
Riesgo de tsunami en caleta Tubul, Región del Biobío: escenarios extremos y transformaciones territoriales posterremoto $^{1}$
}

\author{
Carolina Martínez ${ }^{2}$, Octavio Rojas ${ }^{3}$, Rafael Aránguiz ${ }^{4}$, Arturo Belmonte ${ }^{5}$, \\ Álvaro Altamirano ${ }^{6}$ y Paulina Flores ${ }^{7}$
}

\begin{abstract}
RESUMEN
Se analiza el riesgo de inundación por tsunami para un evento extremo en la localidad de Tubul $\left(37^{\circ} S\right)$, Región del Biobío. Para el análisis de peligrosidad se determinaron tres escenarios asociados a los eventos tsunamigénicos locales de 1835 y 2010 a los cuales se aplicó modelamiento numérico. Se incluyó el evento de 1877 para determinar los efectos de un tsunami lejano. Se analizó la vulnerabilidad en sus dimensiones física, socioeconómica y educativa. El tsunami de 1835 se determinó como evento extremo, con alturas de inundación de $10 \mathrm{~m}$ y cota de inundación de $10 \mathrm{~m}$. Se establecieron niveles de vulnerabilidad altos y medios explicados por la deficiente materialidad de la vivienda y perfil socioeconómico vulnerable de la población, bajos niveles de escolaridad y reacción frente a estos eventos. El riesgo natural obtenido se ubicó en niveles altos para toda la localidad. Se contrastan los resultados con el Plan de Reconstrucción vigente.
\end{abstract}

Palabras clave: Desastres naturales, vulnerabilidad, resiliencia, planificación territorial.

\begin{abstract}
The risk of tsunami inundation is analyzed for an extreme event in the town of Tubul $\left(37^{\circ} \mathrm{S}\right)$, Bio-Bio Region. Three scenarios were identified for risk assessment, in all of which numerical simulation was applied. Two of the three scenarios were local tsunamis, the events of 1835 and 2010, and the event of 1877 was included to determine the effects of a far field tsunami. The tsunami of 1835 was determined as an extreme event, which gave inundation heights of $10 \mathrm{~m}$ and run-up of $10 \mathrm{~m}$. Vulnerability was analyzed from physical, socioeconomic and educational points of views. Two levels of vulnerability were defined, namely medium and high. These levels were selected based on the poor housing materials, the vulnerable socioeconomic profiles of the population, low educational levels and the population's reactions to these tsunami events. The results show that natural risk is obtained at the high level in the whole town. These results were also compared with the current Reconstruction Plan.
\end{abstract}

Key words: Natural Disasters, Vulnerability, Resilience, Territorial Planning

1 Proyecto FONDECYT No 11100379. Artículo recibido el 30 de agosto de 2012, aceptado el 20 de septiembre de 2012 y corregido el 4 de octubre de 2012.

2 Departamento de Geografía, Universidad de Concepción (Chile). E-mail: carolmartinez@udec.cl

3 Centro EULA-Chile y Departamento de Geografía, Universidad de Concepción (Chile). Becario CONICYT 2012. E-mail: ocrojas@udec.cl
4. Departamento de Ingeniería Civil, Universidad Católica de la Santísima Concepción (Chile). E-mail: raranguiz@ucsc.cl

5 Departamento de Geofísica, Universidad de Concepción (Chile). E-mail: abelmonte@dgeo.udec.cl

6 Departamento de Geografía, Universidad de Concepción (Chile). E-mail: alaltamirano@udec.cl

7 Departamento de Geografía, Universidad de Concepción (Chile). E-mail: paulina.flores76@gmail.com 
Los tsunamis son fenómenos recurrentes en la costa chilena debido a su contexto tectónico. La mayoría de estos se generan debido a terremotos de subducción, los cuales presentan un notable registro histórico de efectos devastadores (Lockridge, 1985; Lomnitz, 1970, 1971; Kausel, 1986; Díaz, 1992; Urrutia y Lanza, 1993; Monge, 1993; Lorca y Recabarren, 1994; Lagos, 2000; Palacios, 2012). Recientemente, el terremoto $\mathrm{Mw}=8,8$ del $27 / \mathrm{F}$ de 2010 y posterior tsunami produjeron efectos devastadores en la costa chilena a lo largo de unos $600 \mathrm{~km}$, sin embargo, los más destructivos ocurrieron en las pequeñas localidades costeras de la Región del Biobío, la mayoría de estas dedicadas a la actividad pesquera artesanal. Entre estas, destaca la localidad rural de Tubul, una pequeña caleta de unos 2.000 habitantes, que hasta antes del tsunami tenía como principal actividad la explotación del alga gracilaria (pelillo) que crecía a expensas del humedal Tubul-Raqui. La localidad forma parte de un sistema interconectado de cuencas costeras cuyo contacto con el mar ha formado una extensa llanura litoral con comportamiento de marisma salobre, formando así uno de los humedales costeros más relevantes de la región y que antes del terremoto del 27/F figuraba entre los sitios prioritarios para la conservación y se proyectaba como futuro sitio RAMSAR. El terremoto del 27/F generó en esta localidad un alzamiento costero de 1,4 m que produjo cambios importantes en el nivel de base de los ríos Tubul y Raqui, desecamiento del humedal y pérdida consiguiente de la biodiversidad, así como cambios morfológicos en el sistema estuarial, el cual extendió su zona intermareal en unos $100 \mathrm{~m}$ con estados alternantes entre estuario barrera y lagoon costero (Quezada et al., 2010, Farías et al., 2010; Martínez et al., 2011; Vargas et al., 2011).

Actualmente en la Región del Biobío se desarrolla un proceso de Reconstrucción donde se registran profundas transformaciones territoriales y que hace prioritario contar con estudios científicos aplicables a la toma de decisiones, dado que muchas de las áreas arrasadas por el tsunami están hoy en día siendo repobladas en los mismos lugares afectados e incluso con un aumento de la densificación del uso residencial. Dado que esta localidad fue una de las más afectadas por el tsunami del $27 / F$, y en ella se está eje- cutando un Plan de Reconstrucción que está generando la consolidación residencial y el paso rápido de área rural a urbana, el propósito de esta investigación es analizar el riesgo de tsunami para un evento extremo, que permita generar criterios para el manejo del riesgo, así como al proceso de Reconstrucción que vive actualmente la región.

\section{Materiales y métodos}

\section{Área de estudio}

La localidad de Tubul se localiza en el golfo de Arauco $\left(37^{\circ} 18^{\prime} \mathrm{S}\right)$, y desde el punto de vista administrativo pertenece a la comuna de Arauco; comprende una superficie aproximada de $3 \mathrm{~km}^{2}$ y una población de unos 2.000 habitantes, principalmente concentrada en la ribera del río Tubul (Figura $N^{\circ} 1$ ) y vinculada a la pesca, fundamental actividad económica de la localidad.

El área se inserta en un sistema interconectado de cuencas costeras cuya superficie conjunta es de $274 \mathrm{~km}^{2}$. El clima es de tipo mediterráneo de influencia oceánica con estación seca prolongada y lluvias concentradas en la estación invernal (Devynck, 1970). El sistema se desarrolla sobre la formación Tubul de edad Pliocena (Pineda, 1983), compuesta por depósitos sedimentarios, principalmente areniscas. En el área se reconocen tres niveles de terrazas marinas que fluctúan entre 50 y $100 \mathrm{~m}$. Los procesos eustáticos y tectónicos cuaternarios generaron áreas de relleno y solevantamientos que han influido la morfología del área.

La interacción entre el régimen pluvial de las cuencas costeras y la marea de régimen micromareal ha conformado uno de los humedales más importantes de la región debido a la alta diversidad biológica que lo caracteriza, condición que se ha ido recuperando a dos años de ocurrido el terremoto y tsunami del 27/F de 2010. La asociación SacocornioSpartinentum densiflorae son las especies más relevantes adaptadas a las variaciones de salinidad (Stuardo y Valdovinos, 1989; San Martin et al., 1992; Stuardo et al., 1993; Constabel, 1993; Vásquez, 2009). 
Figura $\mathrm{N}^{\mathrm{o}} 1$

Localización de Tubul, comuna de Arauco, Región del Biobío, Chile.

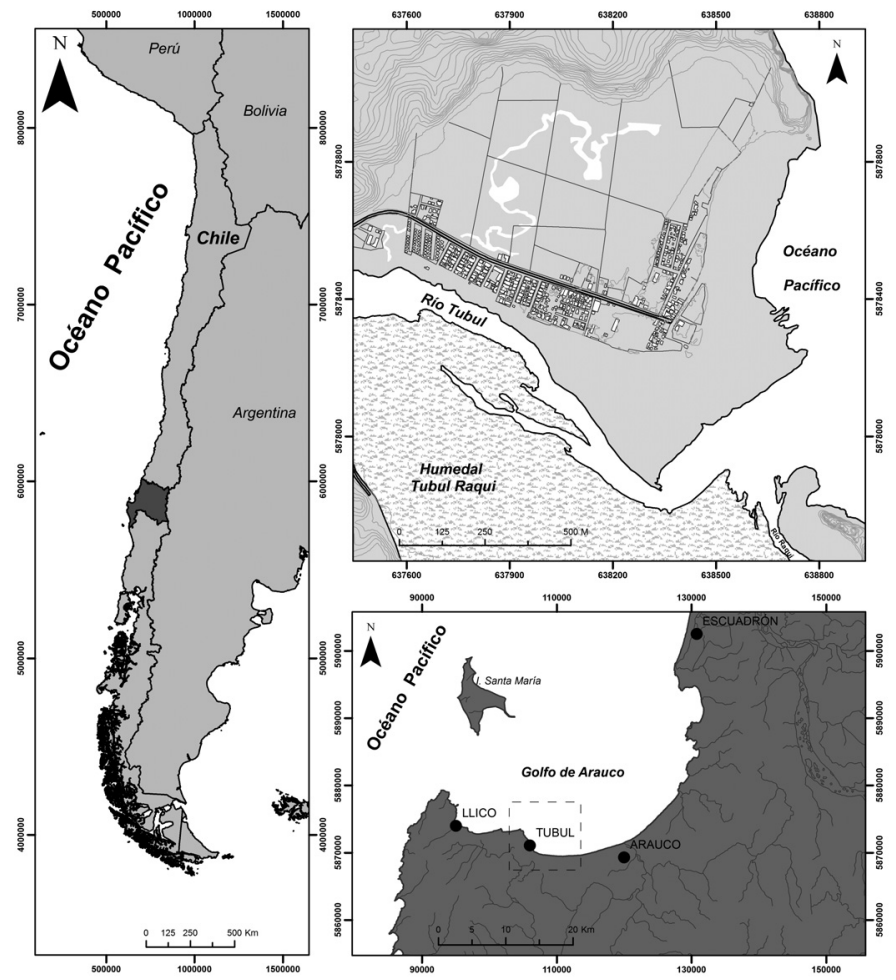

Fuente: Elaboración propia.

\section{Procedimientos}

Para evaluar el riesgo de tsunami en la localidad de Tubul, se definió como la probabilidad de ocurrencia de un peligro natural extremo (tsunami), que puede generar potenciales daños y pérdidas en el medio antrópico, y poner a prueba su capacidad para responder y/o recuperarse (vulnerabilidad) una vez que el peligro se convierte en desastre. De este modo, el riesgo representa la plasmación territorial de una actuación humana poco acorde con las características físico-naturales del territorio donde tiene lugar (modificado de Rojas y Martínez, 2012, en prensa).

El análisis del peligro de inundación por tsunami fue realizado a partir de los resultados de modelamiento numérico aplicando el código NEOWAVE desarrollado en la Universidad de Hawai (Yamazaki et al., 2010). El modelamiento numérico requirió la siguiente secuencia de actividades:

a) Determinación del escenario extremo y de la deformación inicial que genera el tsunami

A través del uso de registros históricos y catálogos sísmicos, se elaboró una base de datos histórica con sismos tsunamigénicos para la zona centro-sur de Chile. Los eventos considerados corresponden a sismos interplaca con magnitudes superiores a $M=8,0$ que generaron tsunamis en la costa de la región. Los eventos seleccionados correspondieron a los de 1835 y 2010. Adicionalmente, se incluyó el evento de 1877 ocurrido en el norte de Chile, con el propósito de establecer los efectos de grandes eventos generados fuera de la región centro-sur pero con impacto potencial en ella, ya que la secuencia histórica de grandes terremotos alojados en la zona 
de ruptura de dicho sismo, permite asignar un periodo de recurrencia de $121 \pm 33$ años (Comte y Pardo, 1991), por lo tanto, un evento de similares características podría repetirse en un futuro cercano.

Los parámetros de falla determinados para calcular la condición inicial para cada tsunami (deformación), fueron aquellos que caracterizan el plano de ruptura y el tamaño del sismo: locación o hipocentro, Magnitud Momento Mw, azimuth (az), manteo (dip), ángulo de deslizamiento (rake), largo (L), ancho (W) y deslizamiento interplaca (D). Estos parámetros fueron deducidos del conocimiento de las características sismo-tectónicas de la región, así como de la relación existente entre la magnitud-momento del sismo, el momento sísmico y las variables área de ruptura y deslizamiento interplaca.

Para definir la condición inicial del tsunami de 1835 no se dispone de mucha información, sin embargo, es posible estimar de manera tentativa la fuente sísmica. Las dimensiones de la fuente fueron estimadas por Nishenko (1985) a partir de datos de inten- sidad registrados en documentos históricos, tales como las observaciones realizadas por Charles Darwin (Gil, 1945). Se define un largo de ruptura de $400 \mathrm{~km}$ y un ancho de 150 $\mathrm{km}$, con un desplazamiento interplaca de 8 m. Del mismo modo, Lomnitz (1970) recoge observaciones sobre el fenómeno que ayudan a localizar de manera aproximada el evento. Por ejemplo, menciona un levantamiento en isla Mocha y en el sector de punta Lavapié, de este modo, la zona de ruptura se puede localizar lo suficientemente al sur como para generar levantamiento en estas zonas, pero no una subsidencia en la desembocadura del río Maule, que también fue descrito. Los parámetros sísmicos utilizados para generar el tsunami de 1835 se muestran en el Cuadro $\mathrm{N}^{\circ} 1$.

Para el caso del tsunami del 27/F de 2010 , se consideraron estos parámetros a partir del registro de alturas de inundación, run-up, tiempos de llegada y dirección de propagación de las ondas del tsunami, considerando la existencia de más de una fuente tsunamigénica. En el caso del evento de 2010, se consideró el modelo de ruptura no homogéneo propuesto por el USGS (Hayes, 2010),

Cuadro $\mathrm{N}^{\circ} 1$

Parámetros de falla para eventos históricos

\begin{tabular}{|l|r|r|}
\hline \multicolumn{1}{|c|}{ Parámetros } & Tsunami 1835 & \multicolumn{1}{|c|}{ Tsunami 1877} \\
\hline Origen (borde norte sobre fosa oceánica) & $35,2^{\circ} \mathrm{S}-73,9^{\circ} \mathrm{W}$ & $19,0^{\circ} \mathrm{S}-72^{\circ} \mathrm{W}$ \\
\hline Dislocación & $8 \mathrm{~m}$ & $12 \mathrm{~m}$ \\
\hline Largo & $400 \mathrm{~km}$ & $420 \mathrm{~km}$ \\
\hline Ancho & $150 \mathrm{~km}$ & $130 \mathrm{~km}$ \\
\hline Rumbo & $\mathrm{N} 15^{\circ} \mathrm{E}$ & $19^{\circ}$ \\
\hline Buzamiento & $18^{\circ}$ & $10 \mathrm{~km}$ \\
\hline Profundidad mínima & $5 \mathrm{~km}$ & $100^{\circ}$ \\
\hline $\begin{array}{l}\text { Angulo } \\
\text { desplazamiento }\end{array}$ & $90^{\circ}$ & 8.8 \\
\hline Magnitud sismo (Escala Richter) & 8.8 & $\mathrm{~N}$ \\
\hline
\end{tabular}

Fuente: Elaboración propia a partir de información histórica. 
el cual ha sido validado regionalmente por Yamazaki \& Cheung (2011), usando registros de las boyas DART 32412, 51406, 32411 y 43412. Este modelo de ruptura utiliza 180 faIlas finitas de largo y ancho fijos, pero con los demás parámetros sísmicos variables.

Para la determinación de los parámetros sísmicos del evento de 1877 registrado en el norte de Chile, se utilizaron las siguientes fuentes principales: mecanismos focales de grandes terremotos como los obtenidos para los eventos de 1995 (Antofagasta) y 2001 (Arequipa), a partir de los cuales es posible estimar los parámetros focales de cada sismo, lo que representa los parámetros del plano de ruptura o plano de Wadati-Benioff (Delouis et al., 2010; Pinares, 2006), y los resultados del estudio asociado al contacto sismogénico interplaca a lo largo de Chile a partir del análisis del conjunto de mecanismos focales asociados a la sismicidad de la región para el periodo 1960-1995 obtenidos de la tesis de grado de Belmonte (1997). Los parámetros sísmicos utilizados en los sismos de 1835 y 1877 se muestran en el Cuadro $N^{\circ} 1$. La Figura $\mathrm{N}^{\circ} 2$ presenta la condición inicial del tsunami para cada uno de los escenarios analizados. b) Elaboración de grillas de diferente resolución espacial

Se generaron cuatro grillas anidadas de diferente resolución espacial $\left(2^{\prime}, 30^{\prime \prime}, 6^{\prime \prime}\right.$ y $\left.1^{\prime \prime}\right)$ con el propósito de aplicar el modelo numérico. Las grillas de menor resolución (2' y $30^{\prime \prime}$ ) utilizaron bases de datos batimétricas internacionales tales como General Bathymetric Chart of the Ocean (GEBCO) y 5-Minute Gridded Global Relief Data Collection (ETOPO-5), mientras que la grilla de resolución 6" se basó en las cartas náuticas del Servicio Hidrográfico y Oceanográfico (SHOA), y la de mayor resolución utilizada en la localidad de Tubul, fue creada utilizando datos batimétricos, topobatimétricos y topográficos de detalle obtenidos en terreno entre 2011 y 2012 mediante GPS TRIMBLE doble frecuencia y ecosonda Garmin acoplado a bote pesquero. Estos datos fueron vinculados a vértices creados especialmente para la zona dado que el terremoto del 27/F de 2010 destruyó la red geodésica regional. Los levantamientos batimétricos cubrieron los ríos Tubul y Raqui, además del sector marino adyacente a la caleta, mientras que los levantamientos topográficos cubrieron la zona estuarial y el humedal hasta la cota de los $20 \mathrm{~m}$. Para cotas mayores se utilizaron datos topográficos

Figura $\mathrm{N}^{\circ} 2$

Condición inicial de los tsunamis 1835, 1877 y 2010
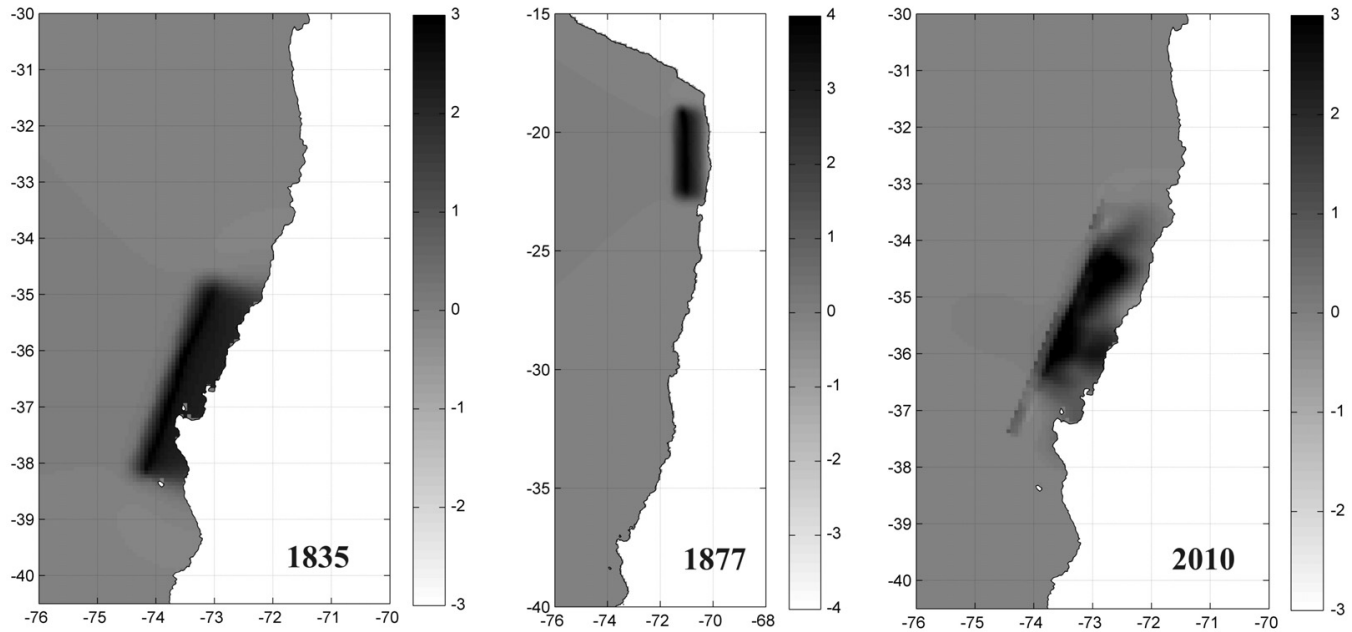

Fuente: Elaboración propia 
extraídos de imágenes AsterGDem, con resolución de $30 \mathrm{~m}$, disponibles en: http://www. gdem.aster.ersdac.or.jp/, generándose contornos cada $5 \mathrm{~m}$.

c) Aplicación del modelo numérico y validación de resultados

La aplicación del modelo de propagación de tsunami consideró los siguientes aspectos: las condiciones iniciales de generación de tsunami aplicadas para deformación estática y dinámica; la batimetría y topografía en región de interés plasmada en cuatro niveles de grillas de tal forma que las menores están contenidas en las mayores; la definición de los tamaños de celda en cada grilla; las constantes físicas del medio y un periodo máximo de propagación e interacción con el entorno variable entre 6 horas para los eventos de campo cercano y 10 horas para el tsunami de campo lejano. Se elaboraron además mareogramas sintéticos para dos sectores de la caleta (en la desembocadura del río y frente a la playa de Tubul) con el propósito de comparar las alturas de ola y los tiempos de arribo del tsunami. Del mismo modo, se definió un mareógrafo sintético en Talcahuano, el cual fue usado para validar los eventos de 1877 y 2010. La Figura № 3 muestra la ubicación de los mareógrafos sintéticos en Tubul y Talcahuano. La Figura $N^{\circ} 3 \mathrm{~A}$ muestra la malla de mayor resolución utilizada para las modelaciones en Tubul, mientras que la Figura $\mathrm{N}^{\circ} 3 \mathrm{~B}$ muestra solo parte de la malla de mayor resolución en la bahía de Concepción.

Tal como se mencionó anteriormente, el modelo de ruptura propuesto por el U.S. Geological Survey (USGS) para el sismo de 2010 ha sido validado regionalmente en el trabajo de Yamazaki \& Cheung (2011), por lo que el presente trabajo realiza una validación local utilizando la información de terreno recogida por los autores durante el postsunami en las diferentes localidades afectadas de la región. Esta información ha quedado registrada en los trabajos de Quezada et al. (2010), Aránguiz (2010), Martínez et al. (2011). Los resultados del modelo numérico en Tubul fueron comparados a su vez con las mediciones realizadas por Fritz et al. (2011) en trabajos de terreno efectuados en la zona. La Figura $\mathrm{N}^{0} 4$ indica las mediciones en la zona en estudio donde el círculo indica la altura de inundación y el triángulo el run-up. Adicionalmente, se realizó una modelación numérica en la bahía de Concepción utilizando la misma resolución de mallas y se comparó el mareógrafo de Talcahuano con un mareógrafo sintético en la misma ubicación (Figura $\mathrm{N}^{\circ}$ 3B).

Por otro lado, para validar el tsunami de 1877 se analizó la serie de tiempo de un mareógrafo virtual en Talcahuano, ya que existen

Figura $\mathrm{N}^{\circ} 3$

Localización de mareógrafos sintéticos
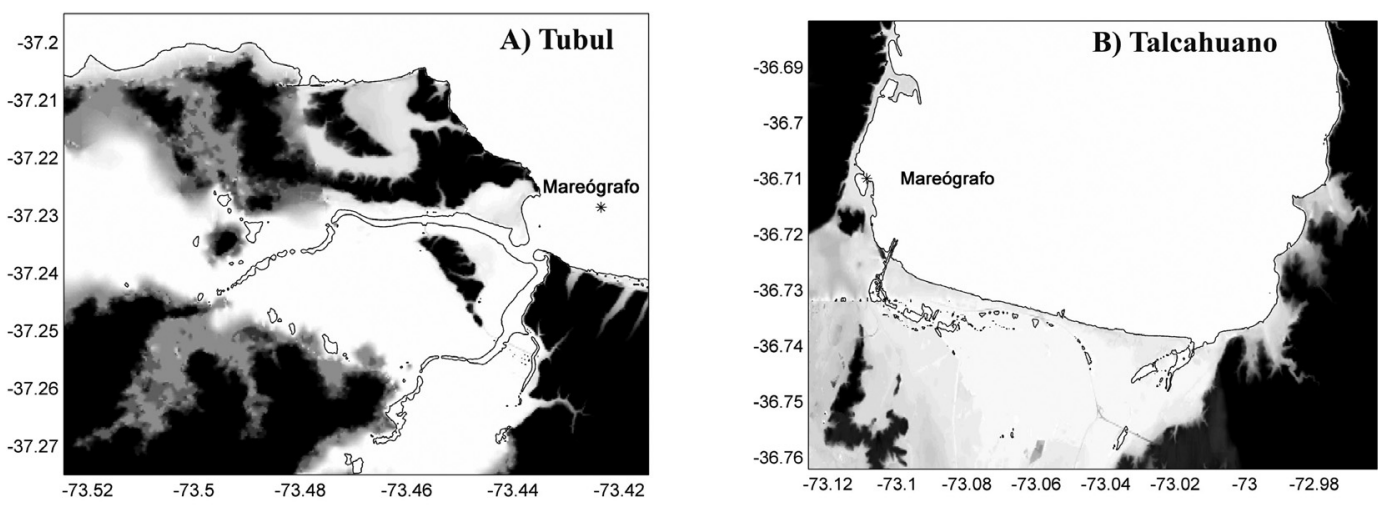

Fuente: Elaboración propia. 
observaciones visuales del tsunami de 1877 en dicha localidad (Soloviev \& Go, 1975). Según estas observaciones, en Talcahuano se observó una oscilación importante del mar unas 3 horas después de ocurrido el sismo. Las observaciones visuales de esa época indican también que en torno a las 0:30 horas (cuatro horas y media después del sismo) el nivel del mar alcanzó 1,2 metros por debajo de la bajamar regular, esto es, unos 1,8 metros aproximadamente, y a las 01:00 horas (5 horas después del sismo) alcanzó los 2 metros sobre la marea máxima regular, esto es, unos 2,8 metros sobre el nivel medio del mar. Finalmente, el parámetro para medir la intensidad y altura de los tsunamis de 1835 y 1877 se realizó a partir de run-up de áreas máximas de inundación en conjunto con valores de series de tiempo.

A partir de los resultados del modelamiento numérico, se elaboró una carta de peligro de inundación por tsunami para la localidad de Tubul, utilizando al SIG ArcGis 9.3, en la cual se incluye el área de propaga- ción máxima asociada al evento extremo. Los niveles de peligrosidad fueron determinados a partir de alturas de flujo de $0,0.5$ y $2.0 \mathrm{~m}$ (Beyer y Aránguiz, 2010).

Para determinar áreas vulnerables frente a tsunami, se definió la vulnerabilidad como el grado de pérdida o grupos de elementos bajo riesgo, resultado de la probable ocurrencia de un suceso desastroso (UNDRO, 1979; Cardona, 1993). Bajo este enfoque, la vulnerabilidad total frente a tsunami (Wilches-Chaux, 1988, en Maskrey, 1993) fue determinada por el estudio de tres vulnerabilidades individuales: física, socioeconómica y educativa. Para su análisis, el poblado fue divido en unidades de análisis (UA) definidas por manzanas derivadas del Plan Regulador Comunal (PRC) de Arauco (Ilustre Municipalidad de Arauco, 2005); las manzanas con dificultades de definición fueron delimitadas con métodos de terreno mediante GPS cartográfico. La división generó 29 UA, que incluyen las aldeas provisorias emplazadas postevento

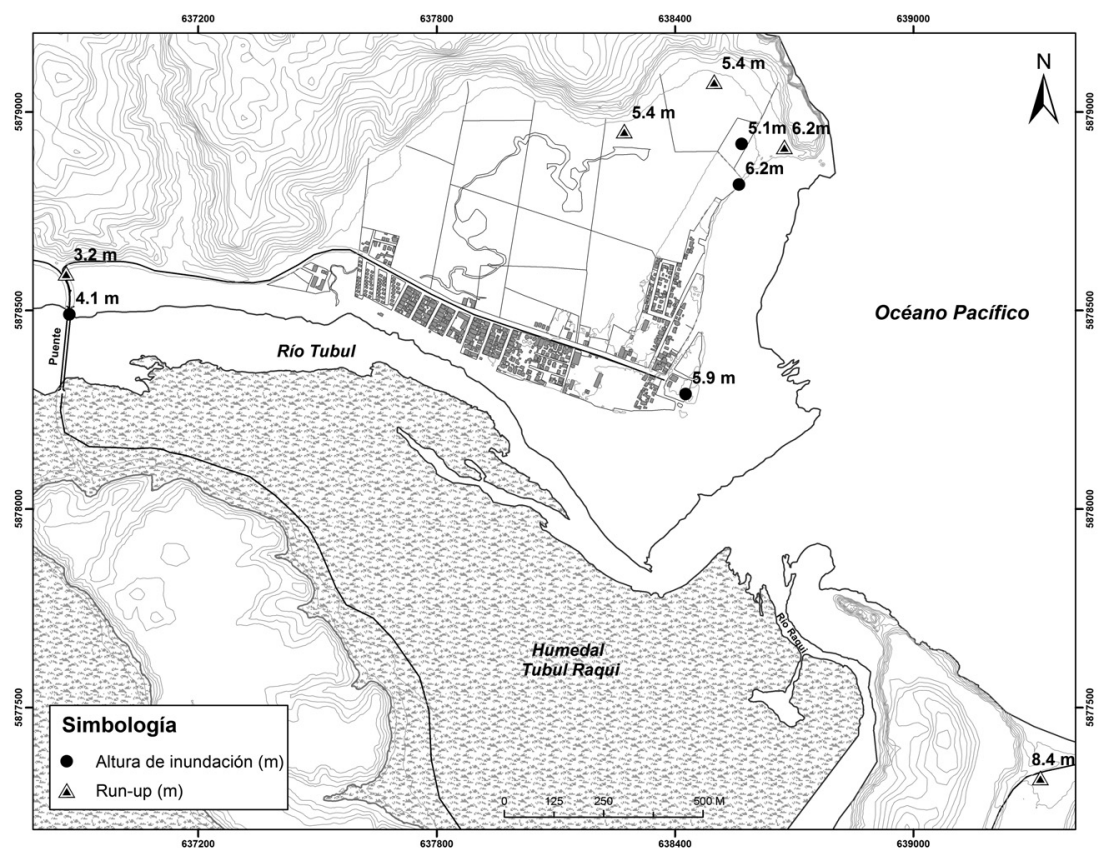

Fuente: Elaboración propia. 
tsunamigénico del 27-F de 2010. La base cartográfica se obtuvo de la Oficina Nacional de Emergencia (ONEMI) Región del Biobío. La población de interés fue definida por los jefes de hogares mayores de 18 años residentes en la aldea de Tubul. El número de la población se determinó en función de datos de la Ilustre Municipalidad de Arauco y se actualizó mediante levantamiento en terreno, que consistió en censar el número de viviendas posterior al tsunami del 27-F, debido a la pérdida de viviendas y su posterior emplazamiento provisorio.

El tamaño muestral se determinó según los siguientes factores: amplitud de universo (494 jefes de hogares); nivel de confianza 95\%; precisión (5\%) y una proporción del $90 \%$, estimada según datos del Ministerio de Planificación para la Región del Biobío (Larrañaga y Herrera, 2010). Aplicada la fórmula para poblaciones finitas, se obtuvo un tamaño de muestra de 109 personas, por razones de pérdidas o rechazo, finalmente se encuestó a 176 sujetos. Para el levantamiento de las variables incluidas en cada vulnerabilidad, se elaboró una encuesta con preguntas cerradas, estructurada según las variables de interés de las vulnerabilidades específicas (Cuadro $N^{\circ} 2$ ). La muestra se aplicó en forma aleatoria simple; el análisis de los datos se realizó en el software InfoStat (Di Rienzo et al., versión 2011).

Para analizar las vulnerabilidades específicas, se utilizó análisis multicriterio (AMC). En la vulnerabilidad física las variables de interés fueron: a) ubicación de la vivienda; b) tipo de vivienda; c) materialidad; d) número de pisos. Para la vulnerabilidad socioeconómica se consideró: a) nivel de ingreso de la pobla- ción; b) actividad económica; c) pobreza. La vulnerabilidad educativa fue determinada en función de: a) nivel de enseñanza; b) nivel de información sobre las causas del fenómeno; y c) reacción frente al fenómeno. Para cada indicador se utilizaron tres niveles de vulnerabilidad: alto, medio y bajo, definidos en función de datos previos. Para determinar áreas jerarquizadas en niveles de vulnerabilidad total frente a tsunami, se evaluaron los resultados de las tres cartografías de vulnerabilidades específicas, según la siguiente ecuación: $C V T=V F+V S E+V E$, donde: CVT (cartografía de vulnerabilidad total); VF (vulnerabilidad física); VSE (vulnerabilidad socioeconómica); VE (vulnerabilidad educativa).

El riesgo final se obtuvo en función de la ecuación $\mathrm{R}=\mathrm{P}^{*} \mathrm{~V}$, donde $\mathrm{R}=$ Riesgo, $\mathrm{P}=$ Peligro, $\mathrm{A}=$ Amenaza. Los factores fueron integrados en una matriz (Eckert et al., 2012; Jalínek et al., 2012), de la multiplicación se obtuvieron tres niveles de riesgo: alto, medio y bajo (Cuadro $\mathrm{N}^{\circ} 2$ ), con puntajes desde 1 a 9 . El nivel de riesgo se aplicó a las unidades de análisis mencionadas en el apartado de vulnerabilidad en un entorno de SIG.

\section{Vulnerabilidad de la caleta de Tubul}

\section{Validación de resultados}

\section{a) Tsunami 1877}

La Figura $\mathrm{N}^{\circ} 5$ indica la serie de tiempo en Talcahuano obtenida para el evento $\mathrm{M}=8.8$ de 1877. Se puede observar una buena correspondencia con las observaciones descri-

Cuadro $\mathrm{N}^{\circ} 2$

Matriz de riesgo de inundación por tsunami, localidad de Tubul

\begin{tabular}{|l|lr|rr|rr|rr|}
\hline \multicolumn{2}{|c|}{ X } & \multicolumn{6}{|c|}{ Peligro } \\
\hline \multirow{4}{*}{ Vulnerabilidad } & & Nivel & & Bajo (1) & & Medio (2) & & Alto (3) \\
\cline { 2 - 9 } & Bajo & $(1)$ & B & $1 \times 1=1$ & B & $1 \times 2=1$ & M1 & X 3 $=3$ \\
& Medio & $(2)$ & B & $2 \times 1=2$ & M & $2 \times 2=4$ & A & $2 \times 3=6$ \\
& Alto & $(3)$ & M & $3 \times 1=3$ & A & $3 \times 2=6$ & A & $3 \times 3=9$ \\
\hline
\end{tabular}

Rango de riesgo: Bajo (1-2), Medio (3-4), Alto (6-9).

Fuente: Elaboración propia. 
tas en Soloviev y Go (1975), pues la primera onda comienza a aumentar después de 3 horas de ocurrido el sismo y luego el nivel desciende a poco más de $2 \mathrm{~m}$ bajo el nivel medio del mar. Además, el máximo nivel alcanzado coincide tanto en la hora como en el valor registrado, esto es, 3 metros, 5 horas después de ocurrido el sismo.

b) Tsunami 2010

La Figura $N^{\circ} 6$ indica la variación del nivel del mar en Talcahuano registrada durante las dos primeras horas del evento y lo obtenido por el modelo numérico. Se puede obser- var que tanto la llegada de las dos primeras ondas como la máxima inundación de la primera son bien representadas. Incluso las dos oscilaciones menores ocurridas entre las dos ondas más grandes son captadas por el modelo numérico. En la Figura $\mathrm{N}^{0} 7$ se presenta el área de inundación medida (izquierda) y modelada (derecha) en Tubul para el evento de 2010, se puede observar una concordancia entre ambas áreas de inundación.

El Cuadro № 3 presenta una comparación de las mediciones realizadas por Fritz et al. (2011) (indicadas anteriormente en la Figura $\left.N^{\circ} 4\right)$ y los valores obtenidos por el modelo

Figura $\mathrm{N}^{\circ} 5$

Serie de tiempo de mareógrafo virtual en Talcahuano para un evento $M=8.8$ frente a Iquique

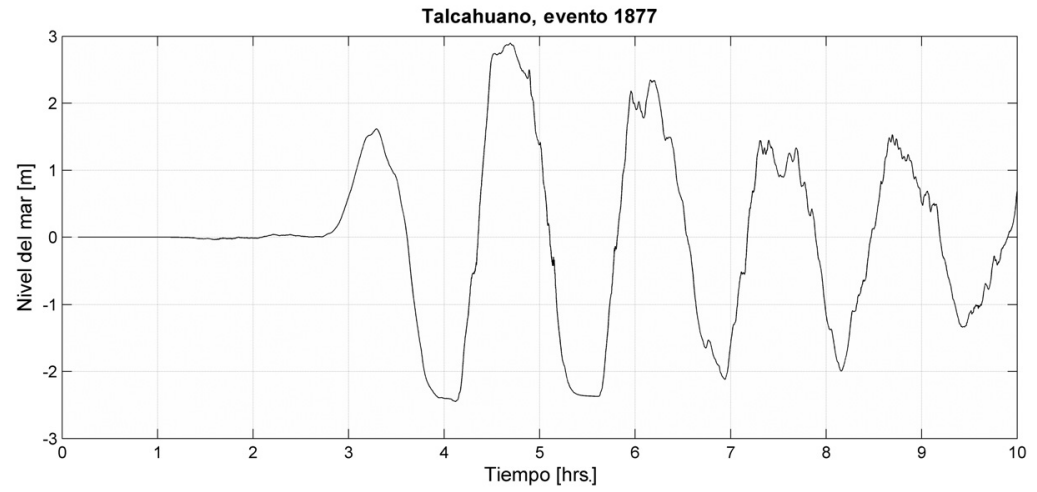

Fuente: Elaboración propia.

Figura $\mathrm{N}^{\mathrm{o}} 6$

Comparación de la variación del nivel del mar medido en Talcahuano y el obtenido en el modelo numérico

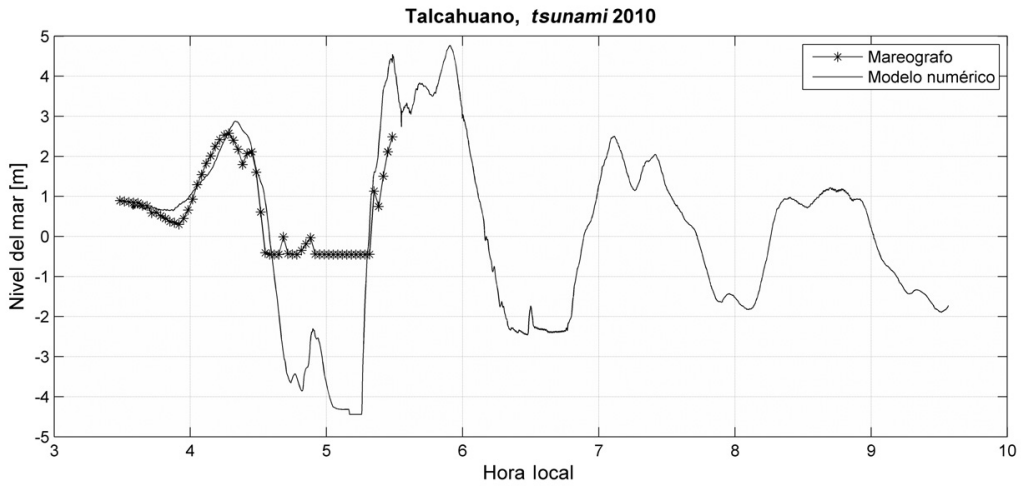

Fuente: Elaboración propia. 
Figura $\mathrm{N}^{\circ} 7$

Área de inundación en Tubul medida (A) y obtenida mediante el modelo numérico (B)
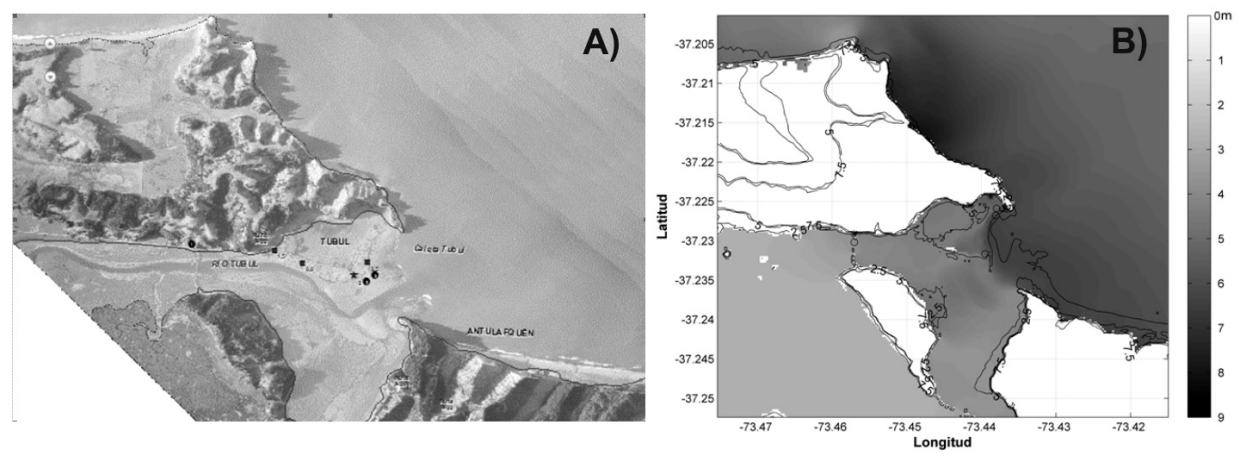

Fuente: A) geoportal.sernageomin.cl/geoportal/rest B) Elaboración propia.

Cuadro $\mathrm{N}^{\circ} 3$

Comparación de resultados modelo numérico y Fritz et al. (2011)

\begin{tabular}{|l|r|r|r|r|l|}
\hline E & N & Medición $(\mathrm{m})$ & Modelo numérico $(\mathrm{m})$ & Diferencia $(\mathrm{m})$ & Tipo \\
\hline$-37,22586$ & $-73,43676$ & 6,2 & 6,13 & 0,07 & runup \\
$-37,22441$ & $-73,43876$ & 5,4 & 5,49 & $-0,09$ & runup \\
$-37,22556$ & $-73,44132$ & 5,4 & 4,47 & 0,93 & runup \\
$-37,22904$ & $-73,45708$ & 3,2 & 3,57 & $-0,37$ & runup \\
$-37,24009$ & $-73,42923$ & 8,4 & 6,50 & 1,90 & runup \\
$-37,22588$ & $-73,43798$ & 5,1 & 5,29 & $-0,19$ & Inundación \\
$-37,22678$ & $-73,43808$ & 6,2 & 6,22 & $-0,02$ & Inundación \\
$-37,23165$ & $-73,43953$ & 5,9 & 5,22 & 0,68 & Inundación \\
$-37,23018$ & $-73,45706$ & 4,1 & 3,77 & 0,33 & Inundación \\
\hline
\end{tabular}

Fuente: Elaboración propia.

numérico. En general, se observa una muy buena correspondencia entre ambos datos, principalmente en la zona plana y desembocadura del río Tubul. Sin embargo, la diferencia más grande se obtiene para el run-up medido de $8.4 \mathrm{~m}$ en la zona este de la desembocadura del río Raqui y no en la caleta de Tubul. Una explicación posible es que el sector donde se midió este valor, posee una topografía en forma de $\mathrm{V}$ que genera concentraciones de energía que el modelo numérico y la resolución de la malla $(\sim 30 \mathrm{~m})$ no son capaces de representar adecuadamente. Para los demás datos se obtuvieron diferencias de centímetros solamente.

\section{Propagación del tsunami}

La Figura $N^{\circ} 8$ indica la serie de tiempo de los mareógrafos virtuales frente a la playa de Tubul para los eventos de 1835 y 2010. En general, se puede observar que ambos eventos muestran un comportamiento similar, a pesar de utilizar fuentes tsunamigénicas distintas, una con modelo de ruptura homogéneo (1835) y la otra con modelo no homogéneo con 180 fallas finitas (2010). Se 
puede observar que si bien se registra un aumento del nivel del mar desde el comienzo del sismo, esta primera onda no es la más destructiva y solo genera un aumento gradual del nivel del mar, durante los primeros 50 minutos, y puede alcanzar los 3 metros en el caso del evento de 1835. Además, la mayor inundación se produce con la tercera onda, que corresponde a una onda de orilla que viaja de norte a sur, la cual ingresa por el extremo norte del golfo de Arauco, la que alcanza 9 metros para el evento de 1835 y 6 metros para el de 2010 .

Del mismo modo, la Figura $N^{\circ} 9$ muestra la variación del nivel del mar en Tubul para el evento de 1877 durante 10 horas. Se pue- de observar que la primera onda alcanza un nivel máximo de 200 minutos (3 horas 20 minutos) después de ocurrido el sismo. Al igual que en el caso anterior, la primera onda no es la más grande, sino las ondas posteriores, las cuales alcanzan un nivel máximo de $2 \mathrm{~m}$.

La Figura № 10 indica el área de inundación máxima y las velocidades de flujo obtenidas del modelo numérico en la localidad para los eventos de 1835, 1877 y 2010 . Se observó que para el evento de 1835, tanto el área de inundación como la velocidad de flujo fueron máximas, alcanzando valores de hasta $10 \mathrm{~m}$ y $7 \mathrm{~m} / \mathrm{s}$, respectivamente. El evento de 2010 presentó características muy similares al de 1835, sin embargo, de efectos

Figura $\mathrm{N}^{\circ} 8$

Serie de tiempo en el mareógrafo virtual frente a Tubul para los eventos de 1835 y 2010

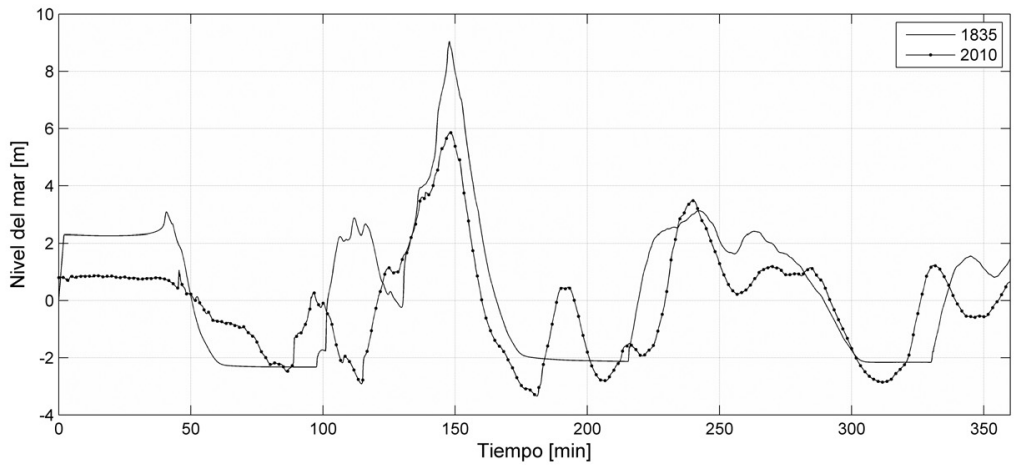

Fuente: Elaboración propia.

Figura $\mathrm{N}^{\circ} 9$

Serie de tiempo en el mareógrafo virtual frente a Tubul para el evento de 1877

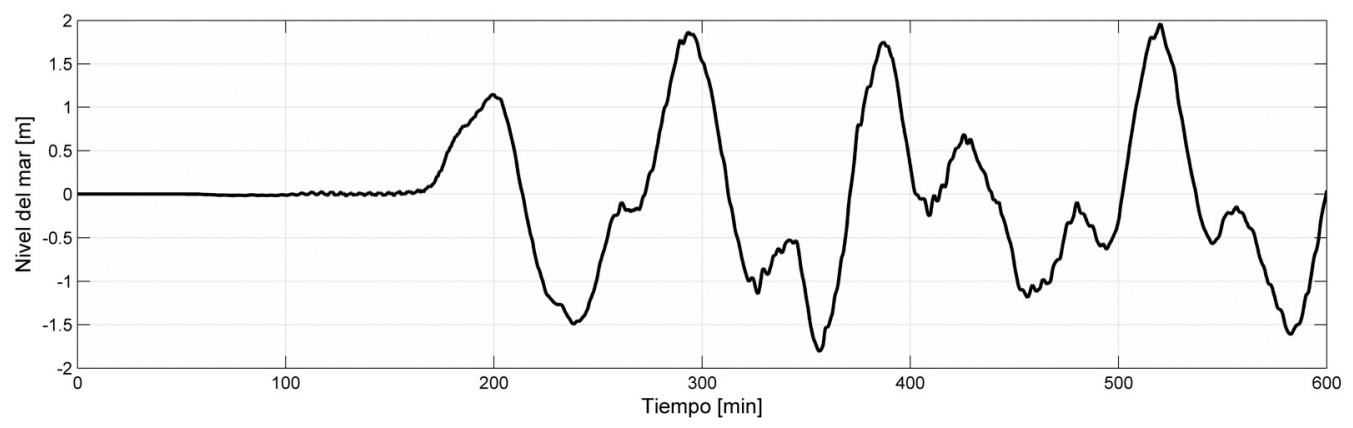

Fuente: Elaboración propia. 
Figura $N^{\circ} 10$

Área de inundación por tsunami y velocidades máximas de flujo

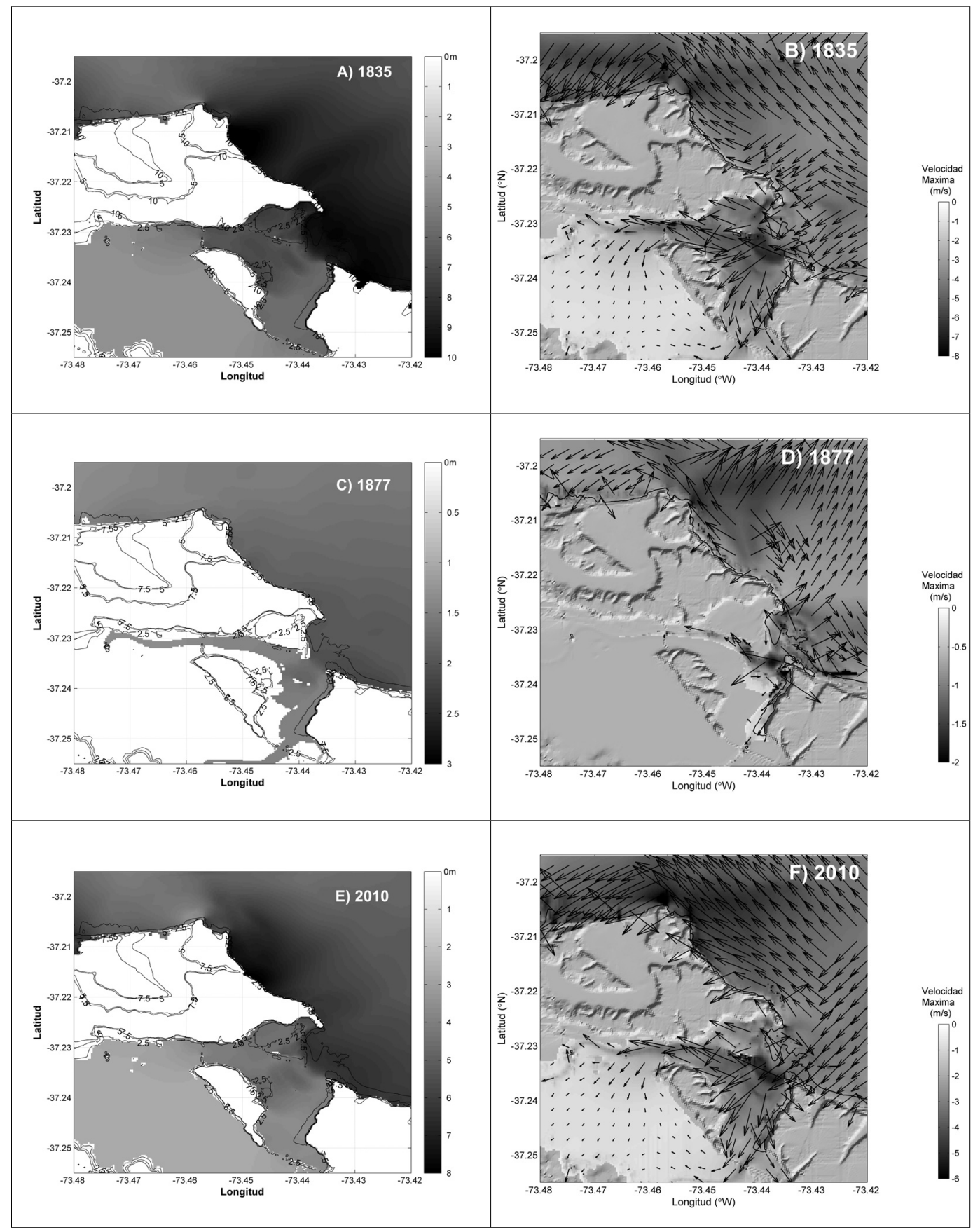

Fuente: Elaboración propia. 
menores dado que la altura máxima de inundación alcanzó los $6 \mathrm{~m}$ en la zona, mientras que las velocidades de flujo registraron hasta $5 \mathrm{~m} / \mathrm{s}$. Para el evento lejano de 1877, la altura de ola máxima fue de $2 \mathrm{~m}$, afectando principalmente al litoral arenoso del área entre Tubul y playa Raqui, con velocidades de flujo de hasta $2 \mathrm{~m} / \mathrm{s}$. La cota de inundación para este evento fue de $2,5 \mathrm{~m}$. Hacia el interior de la localidad, las alturas de ola máximas fueron de 1,7 m. En todos los casos se observó un efecto amortiguador del cerro isla localizado entre los ríos Tubul y Raqui.

La Figura $N^{\circ} 11$ muestra la evolución del tsunami de 1835 a medida que ingresa al golfo de Arauco y se propaga hacia Tubul. La Figura $\mathrm{N}^{\circ} 11 \mathrm{~A}$ corresponde a los primeros 5 min después de ocurrido el evento, y se puede observar claramente que la primera onda que se registra en Tubul (ver Figura $\mathrm{N}^{\circ}$ 8) corresponde a la onda primaria que ingresa al golfo entre la isla Santa María y la península de Arauco, con una dirección hacia el este. Además, se puede observar el efecto de refracción inducido por la presencia del cañón submarino del Biobío, el cual genera un cambio de dirección en la propagación del frente principal, que a su vez es incrementado debido al efecto de difracción de la isla Santa María (Aránguiz, 2012). Las Figuras $N^{\circ}$ $11 \mathrm{C}$ y $11 \mathrm{D}$ muestran la propagación del tsunami desde el tiempo $\mathrm{T}=110$ minutos a 150 minutos, tal que se captura la propagación de la onda de orilla que genera la máxima inundación en Tubul (ver Figura $N^{\circ}$ 8). Se puede observar que esta onda ingresa al golfo de Arauco en dirección norte-sur y en dirección SW a Tubul, lo cual genera un escenario muy desfavorable.

Propagación del tsunami, evento 1835

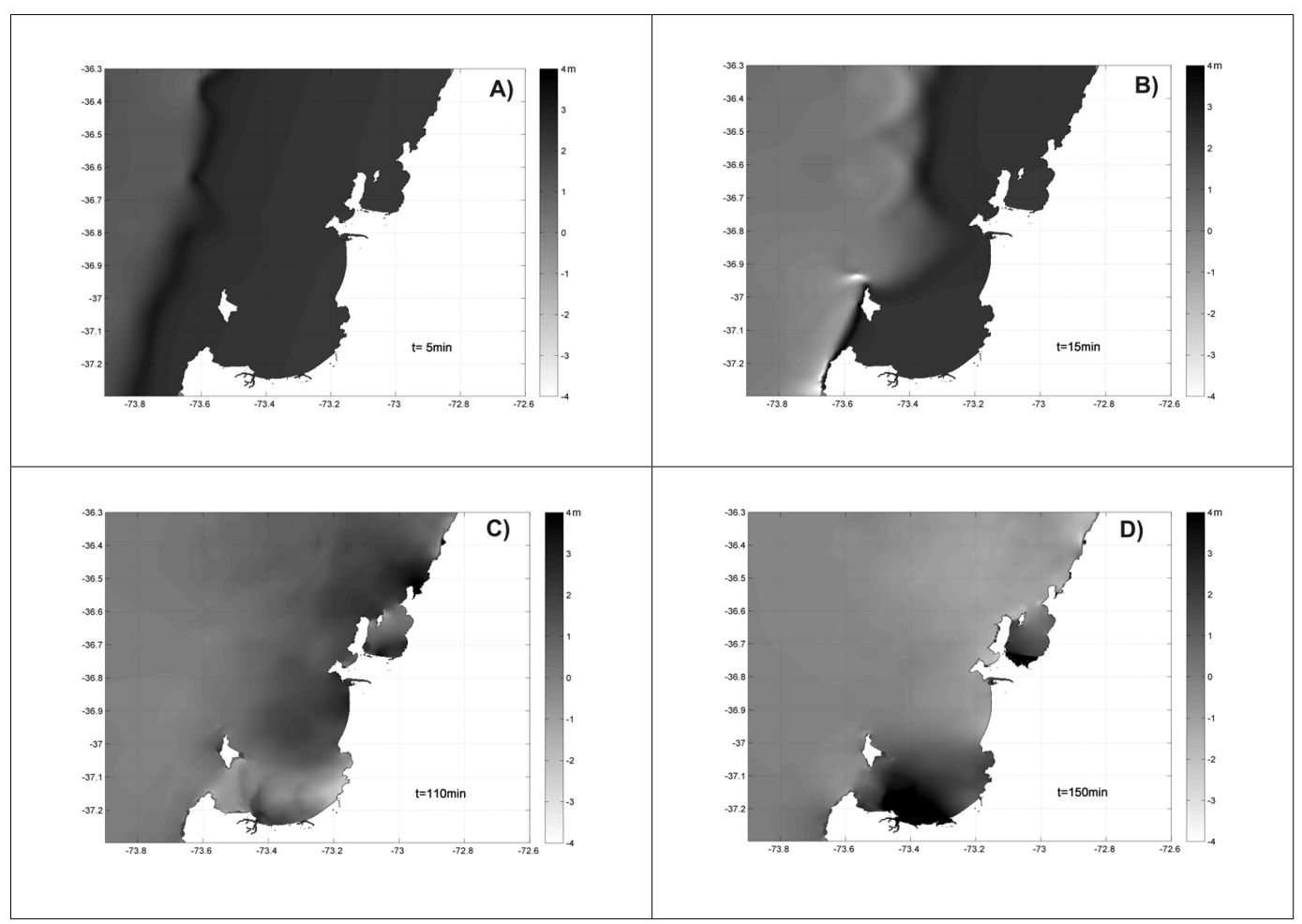

Fuente: Elaboración propia. 
Figura $N^{\circ} 12$

Alturas de inundación y cortes transversales en la zona de Tubul $\left(37^{\circ} \mathrm{S}\right)$, Región del Biobío

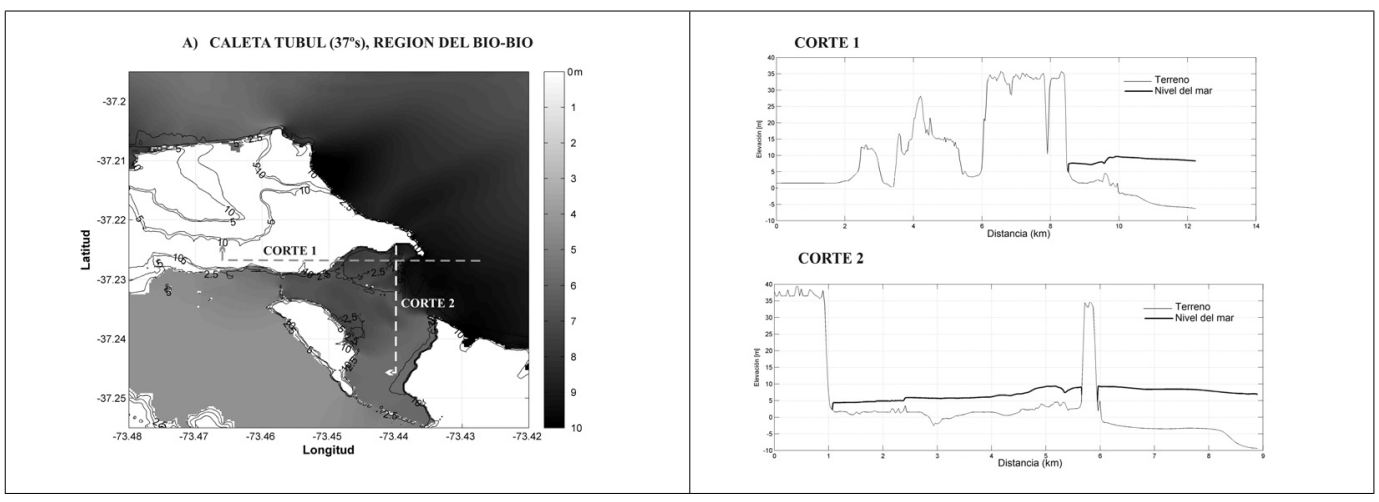

Fuente: Elaboración propia.

\section{Alturas de flujo y niveles de peligrosidad}

La Figura $N^{\circ} 12$ presenta el área de inundación correspondiente al evento de 1835, se indican también dos cortes transversales que permiten visualizar las alturas de inundación y alturas de flujo. Se puede observar que las máximas alturas de flujo en la zona de Tubul superaron los $5 \mathrm{~m}$, pues la elevación del terreno es de 3-4 m, mientras que las alturas de inundación alcanzaron los $10 \mathrm{~m}$.

\section{Niveles de peligrosidad de inundación por tsunami}

Considerando el evento de 1835 como evento extremo, los niveles de peligrosidad fueron obtenidos a partir de las alturas de flujo y campo de velocidades obtenidas del modelo numérico. Dado que el área se comporta por sus características topográficas como una superficie relativamente uniforme, las alturas de inundación alcanzaron la cota de los $10 \mathrm{~m}$, por lo cual toda el área registró una inundación a ese nivel $(>2 \mathrm{~m}$ ) asociadas a velocidades de hasta $8 \mathrm{~m} / \mathrm{s}$.

La superficie con alta peligrosidad correspondió al 66,7\% del área, dentro de la cual se ubica la totalidad del asentamiento de Tubul (Figura $N^{\circ} 13$ ).

\section{Niveles de vulnerabilidad}

La vulnerabilidad física para la localidad presentó solo niveles altos y medios. El nivel medio representó un $58 \%$ de la población; las viviendas construidas se caracterizaron por ser viviendas tipo casa, construidas con material de albañilería y/o madera. En nivel alto se ubicó el $42 \%$ de la población, que reside en viviendas construidas con madera, internit o lata, de solo un piso (Figura $\mathrm{N}^{\circ} 14$ ). En ambos casos el $100 \%$ de las viviendas se encuentra bajo la cota de los 10 m.s.n.m.

La vulnerabilidad socioeconómica de la población de Tubul se concentra en niveles medios y altos. El nivel alto alcanza al 33\% de la población, caracterizándose por presentar bajos salarios y niveles de pobrezaindigencia elevada. La vulnerabilidad media abarca al $64 \%$ de la población. Solamente un $3 \%$ de la población presenta vulnerabilidad socioeconómica baja. En los niveles alto y medio predominaron como actividad económica la extracción de productos del mar; mientras que el nivel bajo corresponde a personas que laboran en el sector terciario, como profesionales y técnicos.

Para la vulnerabilidad educativa se distinguieron niveles altos y medios. La vulnerabilidad media quedó representada por el $79 \%$ de la población; en su mayoría presentaron un nivel de escolaridad que no superó la 


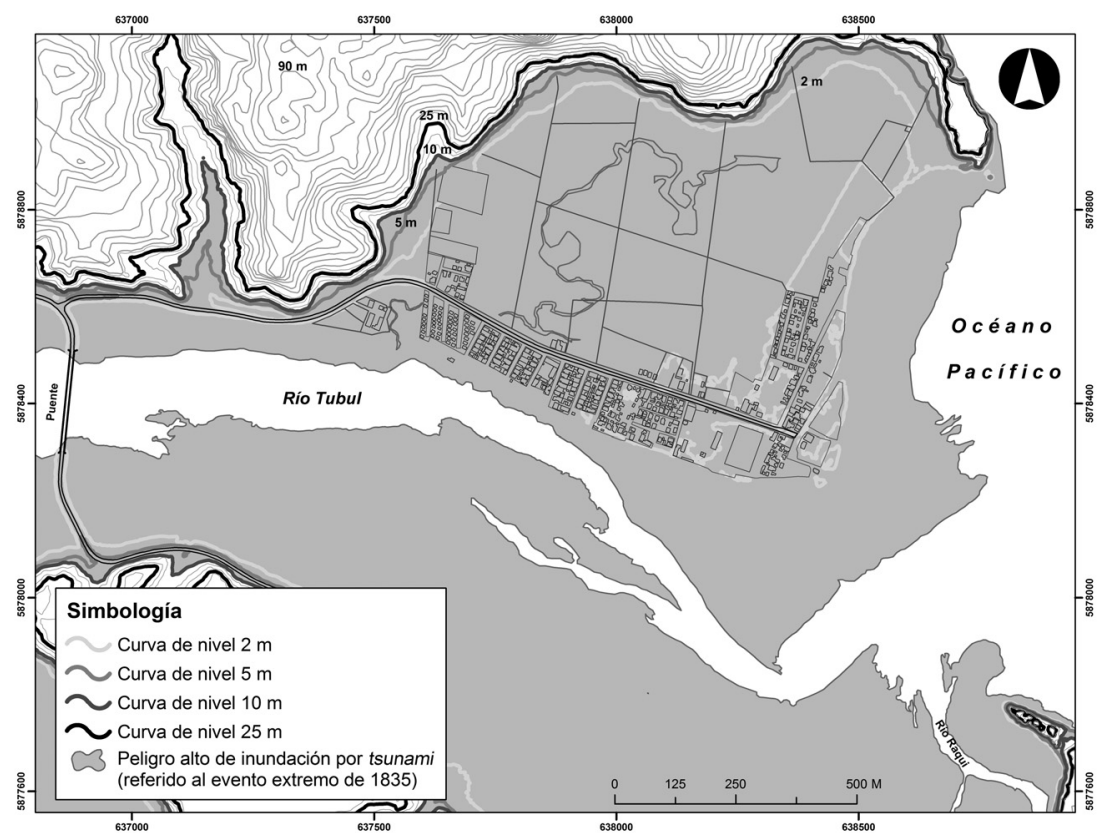

Fuente: Elaboración propia.

enseñanza primaria, su nivel de información sobre las causas de un tsunami supera el $40 \%$, y ante la posibilidad de un evento ellos evacuarán a zonas seguras que no han sido determinadas por instituciones respectivas. En vulnerabilidad alta se encontró el $21 \%$ de la población; este nivel no difirió estadísticamente en cuanto a las características del nivel de instrucción y conocimiento de las causas de un tsunami en comparación a la vulnerabilidad alta; sin embargo, no supieron cómo reaccionar y enfrentar el evento.

Los niveles de vulnerabilidad total de la localidad fueron medios y altos para el $100 \%$ de las unidades de análisis. Estos niveles se explicaron por la exposición de las viviendas, materialidad, nivel de ingreso de la población, escolaridad y reacción frente al fenómeno; la totalidad de estas variables se situaron en rangos de vulnerabilidad medios y altos, explicando la variabilidad de los resultados obtenidos (Figura No 15).

\section{a) Áreas de riesgo por tsunami}

Se determinaron dos niveles de riesgo de inundación por tsunami en la localidad. El nivel alto se registró para el 1,64\% del área de estudio, mientras que el nivel medio para el $65,1 \%$ de la localidad. Este último nivel se registró en las áreas actualmente no ocupadas pero que se localizan dentro del área de peligrosidad de tsunami. Las áreas con alto riesgo incluyen el asentamiento humano de Tubul y corresponden a sectores que registraron una alta vulnerabilidad social sobre áreas de alta peligrosidad de inundación por tsunami (Figura $\left.\mathrm{N}^{0} 16\right)$.

La localidad de Tubul actualmente experimenta un fuerte proceso de urbanización, que de acuerdo al Plan Maestro de Reconstrucción considera ocupar las áreas de riesgo medio y reocupar a través de la entrega de subsidios para la reconstrucción aquellas viviendas destruidas por el terremoto y tsunami del 27/F de 2010. La Figura Nº18 presenta el 
Figura $\mathrm{N}^{\circ} 14$

Materialidad de las viviendas, localidad de Tubul

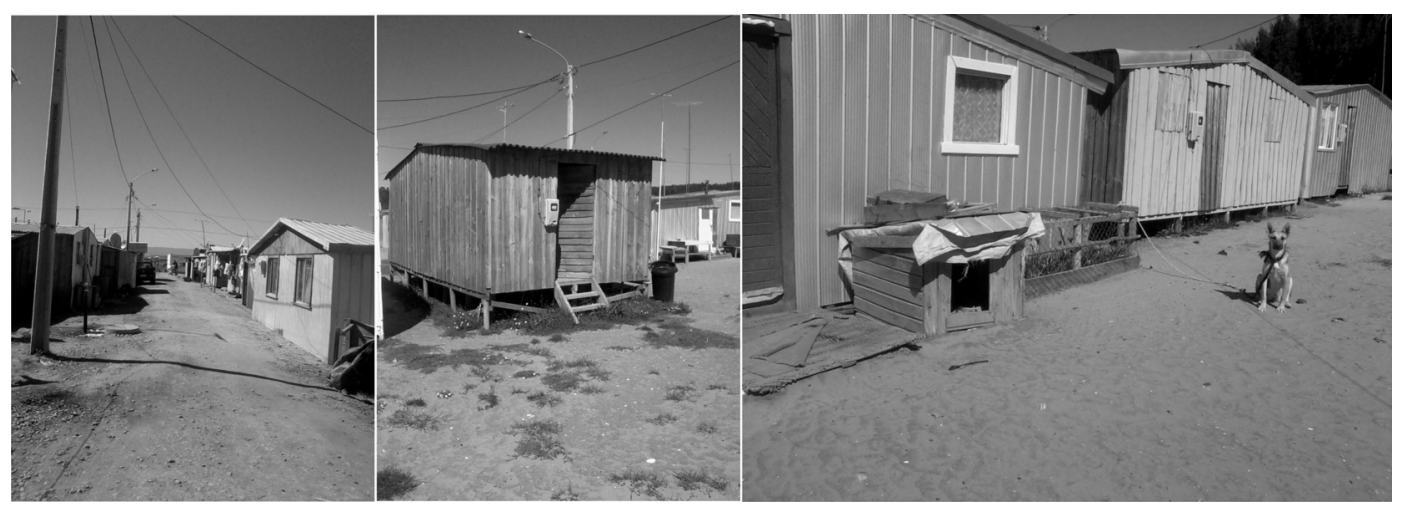

Fuente: Colección personal de los autores.

tipo de viviendas emplazadas dentro de la denominada "zona de viviendas antitsuna$m i^{\prime \prime}$. Este Plan considera un parque de mitigación en el borde costero y la construcción de una costanera con ciclovías.

\section{Conclusiones}

Los efectos diferenciales registrados en la costa del centro sur de Chile, generados por el tsunami del 27F de 2010, establecieron que la configuración de la costa es un aspecto clave a considerar en los escenarios extremos a modelar.

El tsunami del 27F utilizó los cursos locales de las pequeñas localidades costeras (principalmente paleobahías y estuarios) para propagar la energía al interior de estas, generando una mayor superficie inundada. Las características geomorfológicas, en especial de la Región del Biobío, generan condiciones propicias para recibir los impactos de estos eventos dada su herencia morfogenética donde los procesos eustáticos y tectónicos han construido planicies de playa con depósitos de cubierta eólica antigua sobre asentamientos humanos construidos sobre el antiguo delta del Biobío (Isla et al., 2012) o campos dunares que protegieron algunas ciudades costeras de efectos aún mayores como lo fue en Cobquequra, Tirúa y Vegas del Itata. Resulta indispensable conocer estas características de la costa con el propósito de inferir los potenciales efectos de un tsunami extremo.

Algunos rasgos morfológicos mayores tales como cañones submarinos generan cambios en la propagación del tsunami tal como ha sido analizado por Aránguiz (2011 y 2012) para el caso del cañón submarino del Biobío, el cual evidencia un efecto mitigador, a diferencia de otros cañones submarinos en el mundo que generan efectos amplificadores y mayores áreas de inundación (caso Sri Lanka y Blangadesh). Este proceso ya había sido advertido por Quezada (2000) a través del análisis de refracción. De acuerdo con Aránguiz (2011), la localización de la isla Santa María al interior del golfo de Arauco, generaría procesos de difracción, que en conjunto con la reflexión-transmisión en el cañón, tenderían a desviar el frente de onda al lado sur del golfo de Arauco con impacto directo en las localidades de Arauco, Tubul y Llico, lo cual coincidió con las alturas de olas mayores registradas, donde el run-up alcanzó entre 4 y 13 m de acuerdo con observaciones locales (Quezada et al., 2010; Fritz et al., 2011). Los resultados del modelamiento numérico coincidieron con estas mediciones realizadas en terreno y se ajustaron mejor a la zona más expuesta de la bahía de Tubul donde se localiza el asentamiento, que en el sector protegido del río Raqui. Por lo tanto, independientemente de la dirección de 
Figura $\mathrm{N}^{\circ} 15$

Niveles de vulnerabilidad, localidad de Tubul

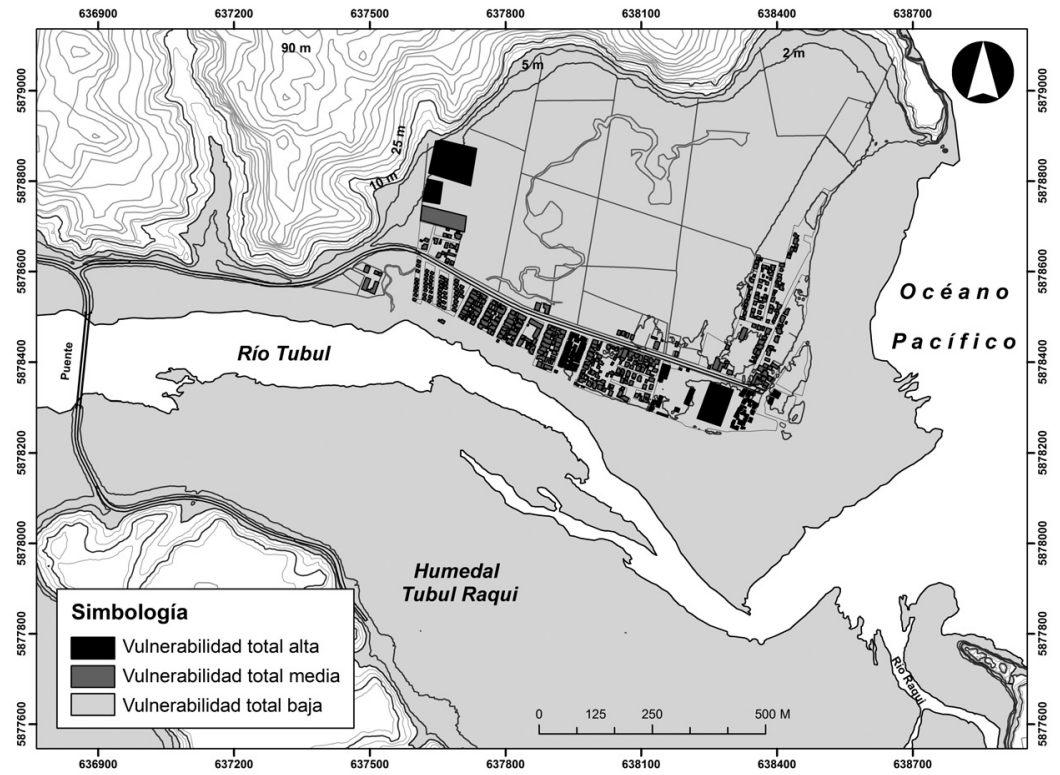

Fuente: Elaboración propia.

Figura $\mathrm{N}^{\circ} 16$

Áreas de riesgo de inundación por tsunami, localidad de Tubul

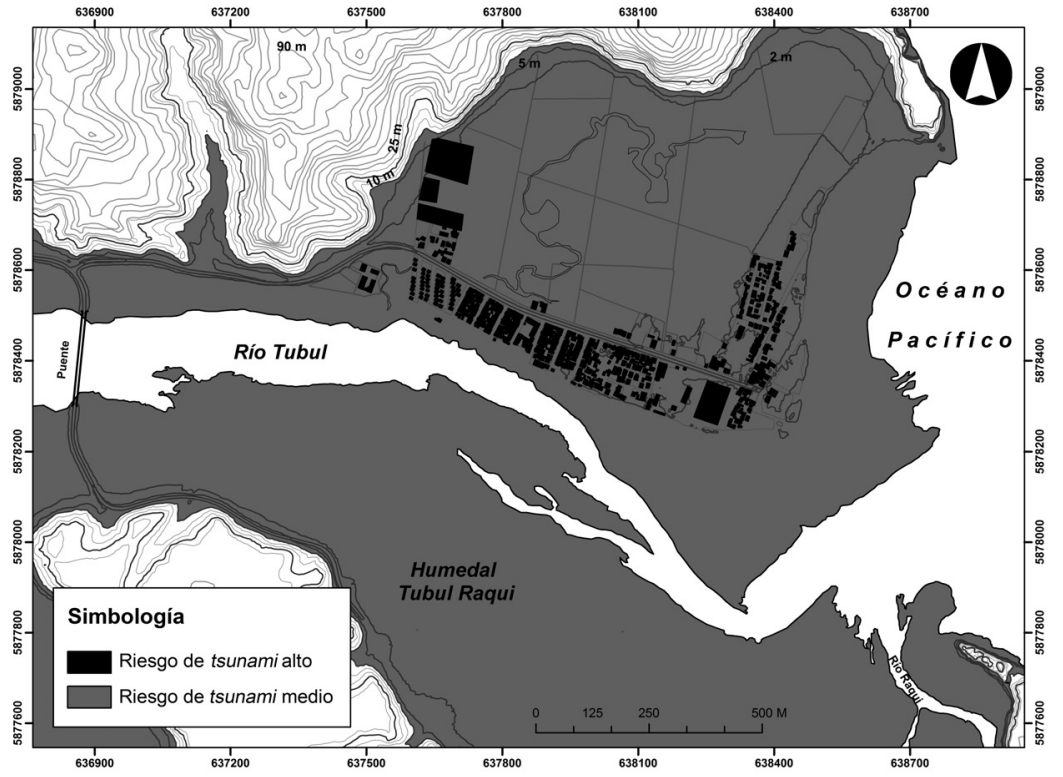

Fuente: Elaboración propia. 
Figura $\mathrm{N}^{\circ} 17$

Plan de Maestro de Reconstrucción posterremoto 27F 2010, localidad de Tubul

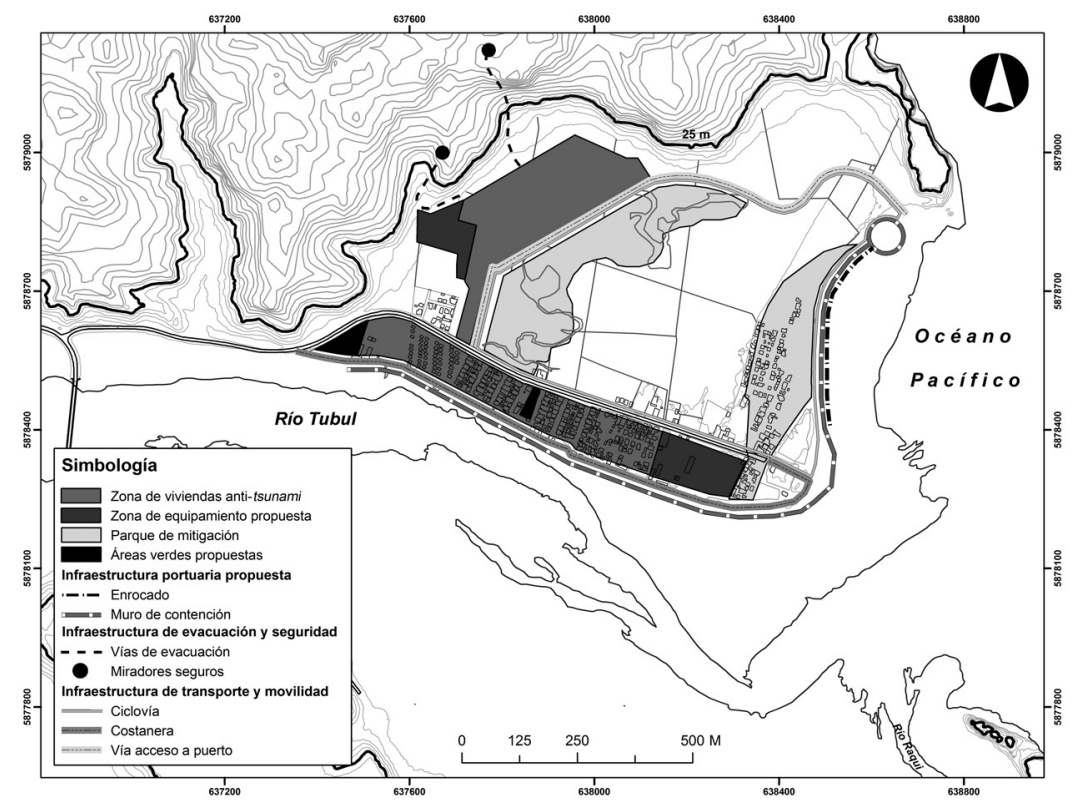

Fuente: Elaboración propia a partir de Plan Maestro de Reconstrucción-Tubul (GORE, 2010)

Figura $N^{\circ} 18$

Tipo de viviendas insertas en el proceso de Reconstrucción en la localidad de Tubul

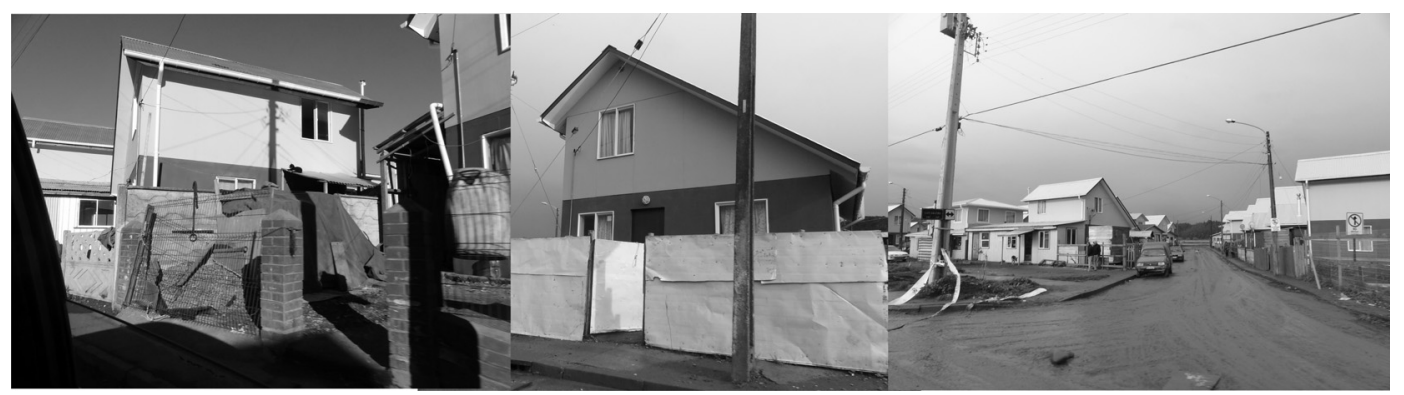

Fuente: Colección personal de los autores.

procedencia del tren de ondas del tsunami, estas localidades estarán más expuestas a los efectos de un evento extremo, como ha sido demostrado con los resultados del modelamiento numérico de los tsunamis de 1835 y 2010, los cuales presentarían similitudes respecto a sus efectos ya que de acuerdo a crónicas históricas el primero produjo los ma- yores daños en bahías expuestas tales como Tirúa, Lebu, Tubul, Llico y Dichato, así también las localidades de Tumbes y la isla Santa María experimentaron alzamientos costeros, en Tubul se determinó en 2 metros (Cisternas et al., 2010), muy cercano al establecido para el terremoto de 2010 de 1,4 metros (Quezada et al., 2010; Farías et al., 2010). 
Por otro lado, se sabe que el terremoto del $27 \mathrm{~F}$ de 2010 generó cambios morfológicos drásticos en la costa de la Región del Biobío, con alzamientos costeros de $1,4 \mathrm{~m}$ en Tubul y $1,7 \mathrm{~m}$ en Lebu, situación que afectó a localidades ubicadas al sur del golfo de Arauco (Quezada et al., 2010), lo que ha generado una nueva realidad geográfica que debe incluirse al momento de efectuar modelamiento numérico. Esta nueva realidad, en conjunto con las transformaciones territoriales que actualmente experimentan las localidades afectadas por el sismo-tsunami del 27F, hace que permanentemente se deba modelar realidades dinámicas que hacen que tanto la peligrosidad como la vulnerabilidad cambien en el tiempo. Tubul es, actualmente, una de las localidades más intervenidas producto de la Reconstrucción y se ha observado, solo en el último año, un rápido proceso de urbanización en áreas de alta peligrosidad, así también se proyecta la construcción de una costanera y ciclovía dentro de la zona intermareal, donde eventos comunes en el área tales como marejadas y olas de tormentas tienen la capacidad de producir cambios morfológicos. Uno de los principales factores que elevan la vulnerabilidad por tsunami en Tubul es el tipo de vivienda y su materialidad, localizadas al borde de la zona intermareal y dispuestas en parcelas irregulares, estas viviendas, construidas en madera, de un piso y materiales ligeros, pueden ser masivamente arrasadas cuando el tsunami desarrolla velocidades de corrientes del orden de los $2 \mathrm{~m} / \mathrm{s}$ y alturas de flujo o profundidad de la inundación sobre los 2 m; así, experiencias mundiales con eventos extremos han establecido una destrucción total de las viviendas con alturas de flujo cercanas a $5 \mathrm{~m}$ y velocidades de las corrientes de $4 \mathrm{~m} / \mathrm{s}$ (Lagos et al., 2008). Los resultados del modelamiento numérico establecieron alturas de ola de $9 \mathrm{~m}$ para el evento de 1835 y 6 m para el de 2010, mientras que las velocidades de las corrientes fueron de $8 \mathrm{~m} / \mathrm{s}$ para el evento de 1835 y de $6 \mathrm{~m} / \mathrm{s}$ para 2010, lo cual elevó el riesgo debido a que la localidad está emplazada en áreas de alta peligrosidad de inundación por tsunami.

Por otro lado, la caleta, históricamente, surge vinculada estrechamente a la actividad pesquera y en especial a la recolección del alga gracilaria, esta última explotada casi ex- clusivamente por mujeres. Estas actividades, aunque no habían causado impacto en los indicadores socioeconómicos, dado que en la localidad el nivel de pobreza ha sido históricamente alto y vinculado a otros problemas sociales, el terremoto y tsunami de 2010 generó un aumento en las características vulnerables de la población (Altamirano, 2011), que hoy contrastan con una visión de ocupación del territorio que está fuera de la manera idónea de gestionar las áreas de riesgo ya que se está priorizando el emplazamiento de infraestructura urbana por sobre las necesidades que vinculan a la población con su espacio natural (arraigo e identidad), que es la pesca y el uso de recursos naturales tales como la recolección de algas. La caleta Tubul en este sentido se localiza en uno de los principales humedales de la región, hoy en día en lenta recuperación (Valdovinos 2012), y ocupa la antigua planicie de playa construida por procesos fluviomarinos y tectónicos sobre la cual se desarrolla la marisma salobre, por lo cual es una zona frágil y que requiere de manejo ambiental. El proceso de Reconstrucción en este sentido, debe considerar la naturaleza y fragilidad de los sistemas costeros en donde se interviene, con el fin de evitar la degradación ambiental de estos y consecuencias negativas ante futuras situaciones de riesgo de desastre, que como se ha demostrado los fenómenos de tsunamis son eventos recurrentes en la costa chilena y en especial en la región del Biobío (Lagos, 2000; Palacios, 2012).

Los estudios de riesgo hoy en día enfatizan en los conceptos de adaptación y resiliencia a partir de las lecciones aprendidas de eventos extremos en diferentes partes del mundo (Paton \& Johnston, 2006; Cutter et al., 2008; Jabareen, 2012). Se hace necesario, por lo tanto, incluir e incorporar a la forma de ocupar el territorio, caracterizado por una intensa antropización de la zona costera, la extensa recurrencia histórica de estos fenómenos y la determinación de escenarios extremos, con el fin de disminuir o controlar la situación de desastres que estos provocan.

\section{Referencias bibliográficas}

ALTAMIRANO, A. Análisis de vulnerabilidad por tsunami en la localidad de Tubul, Región del Bio-Bío, Chile. Concepción: Tesis para 
optar al Título de Geógrafo, Universidad de Concepción, 2011 (Inédito).

ARÁNGUIZ, R. Modelación numérica del tsunami de 2010 en la Bahía de Concepción utilizando deformación estática no uniforme. Obras y Proyectos, 2010, № 8, p. 12-18

ARÁNGUIZ, R. Estudio preliminar sobre el efecto del cañón submarino del Biobío en la propagación de tsunamis. En: Sociedad Chilena de Ingeniería Hidraúlica, XX Congreso Chileno de Hidráulica, Santiago de Chile, 2011. Disponible en Internet: http://cimp.ucsc.cl/wpcontent/uploads/2011/10/Aranguiz.pdf

ARÁNGUIZ, R. Effect of a submarine canyon on tsunami propagation in the Gulf of Arauco, Chile. In: International Conference on Coastal Engineering, Santander, 2012.

BELMONTE, A. Análisis del Contacto Sismogénico Inter-Placa a lo largo de Chile. Santiago de Chile: Tesis para optar el Título de Ingeniero Civil Mención Estructuras y Magíster en Ciencias Mención Geofísica, Universidad de Chile, 1997 (Inédito).

BEYER, J. y ARÁNGUIZ, R. Estudios de Riesgos de Sismo y Maremoto para las comunas costeras de la región del Bio-Bío. Concepción: Centro de Investigación Marítimo Portuario, Universidad Católica de la Santísima Concepción (UCSC), 2010 (Inédito).

CARDONA, O. Evaluación de la amenaza, la vulnerabilidad y el riesgo: Elementos para el Ordenamiento y la Planeación del Desarrollo. La Red, 1993. Disponible en Internet: http:// www.desenredando.org/public/libros/1993/ Idnsn/html/cap3.htm

CISTERNAS, M.; MELNICK, D.; ELY, L.; WESSON, R. \& NORAMBUENA, R. Similarities between the great Chilean earthquakes of 1835 and 2010. American Geophysical Union Chapman Conference, 2010, p. 19.

COMTE, D. \& PARDO, M. Reappraisal of great historical earthquakes in the northern Chile and southern Peru seismic gap. Natural Hazards, 1991, Vol. 4, p. 23-44, doi:10.1007/ BF00126557.

CONSTABEL, S. Ambientes sedimentarios de los estuarios Tubul y Raqui, VIII Región, Chi- le. Concepción: Tesis para optar al Grado de Doctor en Ciencias Ambientales, Universidad de Concepción, 1993 (Inédito).

CUTTER, S.; BARNES, L.; BERRY, M.; BURTON, C.; EVANS, E.; TATE, E. \& WEBB, J. A place-based model for a understanding community resilience. Global Environmental Change, 2008, № 18, p. 598-606.

DELOUIS, B.; NOCQUET, J. \& VALEÉ, M. Slip distribution of the February 27, 2010 $M w=8.8$ Maule Earthquake, central Chile. From static and high-rate GPS, InSAR and teleseismic data. Geophysical Research Letters, 2010, No 37, p. L17305.

DEVYNCK, J. Contribución al estudio de la circulación atmosférica en Chile y el clima de la región del BioBío. Concepción: Memoria de Título para optar al título de Meteorólogo, Universidad de Concepción, 1970 (Inédito).

DíAZ, J. Estudio de fuentes de tsunamis y de terremotos: aplicación en el Norte de Chile y Sur de Perú. Valparaíso: Memoria para optar al Título de Oceanógrafo, Pontificia Universidad Católica de Valparaíso, Chile, 1992 (Inédito).

Di RIENZO, J.; CASANOVES, F.; BALZARINI, M.; GONZÁLEZ, L.; TABLADA, M. y ROBLEDO, C. InfoStat versión 2011. Córdoba: Grupo InfoStat, FCA, Universidad Nacional de Córdoba, Argentina. Disponible en internet: http://www.infostat.com.ar

ECKERT, S.; JELINEK, R.; ZEUG, G. \& KRAUSMANN, E. Remote sensing-based assessment of tsunami vulnerability and risk in Alexandria, Egypt. Applied Geography, 2012, Vol. 32, No 2, p. 714-723.

FARÍAS, M.; VARGAS, G.; TASSARA, A.; CARRETIER, S.; BAIZE, S.; MELNICK, D. \& BATAILLE, K. Land-level changes produced by the $2010 \mathrm{Mw}$ 8.8 Chile earthquake. Science, 2010, Vol. 329, p. 916.

FRITZ, H.; PETROFF, C.; CATALÁN, P.; CIENFUEGOS, R.; WINCKLER, P.; KALLIGERIS, N.; WEISS, R.; BARRIENTOS, S.; MENESES, G.; VALDERAS-BERMEJO, C.; EBELIG, C.; PAPADOPOULOS, A.; CONTRERAS, M.; ALMAR, R.; DOMÍNGUEZ, J. \& SYNOLAKYS, C. Field 
Survey on the 27 February 2010 Chilean Tsunami. Pure and Applied Geophysics, 2011, № 168, p. 1989-2010.

GIL, J. Charles Darwin, viaje de un naturalista alrededor del mundo. Buenos Aires: El Ateneo, 1945.

GOBIERNO REGIONAL, REGIÓN DEL BÍO-BÍO. Plan de Reconstrucción del Borde Costero, PRBC-18. Concepción: Plan Maestro Tubul, 2010. Disponible en Internet: http:// www.minvu.cl/opensite_20101207193158. aspx

HAYES, G. Finite fault model, updated result of the feb 27, $2010 \mathrm{Mw} \mathrm{8,8} \mathrm{Maule} \mathrm{Chile}$ Earthquake. National earthquake Information Center (NEIC) of United States Geological Survey 2010. Disponible en Internet en:

http://earthquake.usgs.gov/earthquakes/ eqinthenews/2010/us2010tfan/finite_fault1.php

ILUSTRE MUNICIPALIDAD DE ARAUCO. Plan Regulador Comunal, 2005. Concepción. Disponible en Internet en: http://www.muniarauco.cl/tpma/plan_regulador.html

ISLA, F.; QUEZADA, J.; MARTÍNEZ, C.; FERNÁNDEZ, A. \& JAQUE, E. The evolution of the BíoBío delta and the coastal plains of the Arauco Gulf, BíoBío Region: the Holocene sea-level curve of Chile. Journal of Coastal Research, 2012, № 28, p. 102-111.

JABAREEN, Y. Vulnerability of cities to extreme space weather events: A new frontier of a multidisciplinary urban research. Natural Science, 2012, Vol. 4, No 6, p. 368-371.

JELÍNEK， R.; KRAUSMANN， E.; GONZÁLEZ, M.; ÁLVAREZ-GÓMEZ, J.; BIRKMANN, J. \& WELLE, T. Approaches for tsunami risk assessment and application to the city of Cádiz, Spain. Natural Hazards, 2012, Vol. 60, No 2, p. 273-293.

KAUSEL, E. Los terremotos de agosto de 1868 y mayo de 1877 que afectaron al sur de Perú y norte de Chile. Boletín de la Academia Chilena de Ciencias, 1986, Vol. 3, № 1, p. 8-13.
LAGOS, M. Tsunamis de origen cercano a las costas de Chile. Revista de Geografía Norte Grande, 2000, № 27, p. 93-102.

LAGOS, M.; CISTERNAS, M. y MARDONES, M. Construcción de viviendas sociales en áreas de riesgo de tsunami. Revista de la Construcción, 2008, Vol. 7, № 2, p. 4-16.

LARRAÑAGA, O. y HERRERA, R. Encuesta Post Terremoto: Principales resultados. Efectos en la calidad de vida de la población afectada por el terremoto/tsunami. Santiago de Chile: MIDEPLAN, 2010.

LOCKRIDGE, P. Tsunamis in Chile-Perú. Boulder: Report SE-39 Worl Data Centera for solid earth and geophysics, 1985.

LOMNITZ, C. Major earthquakes and tsunamis in Chile during the period 1535 to 1955. Soderdruck aus der Geologischen Rundschau Band, 1970, № 59, p. 938-690.

LOMNITZ, C. Terremotos y Tsunamis en Chile. Revista Geofísica Panamericana, 1971, No 1, p. 151-178.

LORCA, E. y RECABARREN, M. Terremotos y Tsunamis o Maremotos. Santiago de Chile: Comisión Oceanográfica Intergubernamental, Centro Internacional de Informaciones, 1994.

MARTÍNEZ, C.; ROJAS, O.; JAQUE, E.; QUEZADA, J.; VÁSQUEZ, D. y BELMONTE, A. Efectos territoriales del tsunami del 27 de Febrero de 2010 en la costa de la Región del Bio-Bío. Revista Geográfica de América Central, Número Especial EGAL, 2011 Costa Rica, p. 1-16.

MASKREY, A. Vulnerabilidad y mitigación de desastres. En: MASKREY, A (Compilador). Los Desastres No Son Naturales. La Red, 1993. Disponible en internet: http://www.desenredando.org/public/revistas/dys/rdys03/dys3-1.0oct-24-2001-TODO.pdf

MONGE, J. Estudios de riesgo de tsunami en costas chilenas. Jornadas Chilenas de Sismología e Ingeniería Antisísmica, 1993, № 2, p. 3-22.

NISHENKO, S. Seismic potential for large and great interplate earthquakes along the chilean and southern peruvian margins of south 
america: a quantitative reappraisal. Journal of Geophysical Research, 1985, No 90, p. 3589-3615.

PALACIOS, A. Sismicidad histórica de la ciudad de Concepción desde su fundación en 1550 hasta su traslado en 1751. Santiago de Chile: Servicio Nacional de Geología y Minería, Boletín Nº 64, 2012.

PATON, D. \& JOHNSTON, D. Disaster Resilience, An Integrated Approach. Illinois: Charles C. Thomas Publisher Ltda., 2006.

PINARES, P. Estudio de la distribución espacial y temporal de la sismicidad en el norte de Chile. Santiago de Chile: Tesis para optar al título de Ingeniero Civil, Facultad de Ciencias Físicas y Matemáticas, Universidad de Chile, 2006 (Inédito).

PINEDA, V. Evolución paleogeográfica de la Península de Arauco durante el Cretácico Superior-terciario. Concepción: Memoria de Título para optar al Título de Geólogo, Universidad de Concepción, Chile, 1983 (Inédito).

QUEZADA, J. Peligrosidad de tsunamis en la zona de Concepción. Congreso Geológico Chileno, № 9, 2000, p. 92-96.

QUEZADA. J.; JAQUE, E.; BELMONTE, A.; FERNÁNDEZ, A.; VÁSQUEZ, D. y MARTÍNEZ, C. Movimientos cosísmicos verticales y cambios geomorfológicos generados durante el terremoto $\mathrm{Mw}=8,8$ del 27 de Febrero de 2010 en el centro-sur de Chile. Revista Geográfica del Sur, 2010, Vol. 1, № 2, p. 11-45.

ROJAS, O. y MARTÍNEZ, C. Riesgos Naturales: evolución y modelos conceptuales. Revista Universitaria de Geografía, 2012 (en prensa).

RUEGG, J.; OLCAY, Y. \& LAZO, D. Co, post and pre-seismic displacements associated with 1995 July $30 \mathrm{Mw}=8.1$ Antofagasta, Chile, earthquake as constrained by InSAR and GPS observations. Seismological Research Letters, 2011, № 72, p. 673-678.

SAN MARTÍN, C.; CONTRERAS, D.; SAN MARTÍN, J. y RAMÍREZ, C. Vegetación de las marismas del centro-sur de Chile. Revista Chilena de Historia Natural, 1992, № 65, p. 327-342.
STUARDO, J.; VALDOVINOS, C.; FIGUEROA, R. y OCHIPINTI, A. Los ambientes costeros del Golfo de Arauco y área adyacente. Concepción Serie Monografías Científicas, Centro EULA, 1993, p. 31-43.

STUARDO, J. y VALDOVINOS, C. Estuarios y lagunas costeras: ecosistemas importantes de Chile central. Revista Ambiente y Desarrollo, 1989, No 5, p. 107-115.

SOLOVIEV, S. \& GO, Ch. A catalogue of tsunamis on the Eastern shore of the Pacific Ocean. Moscow: Nauka Publishing House, 1975.

UNITED NATIONS DISASTER RELIEF ORGANIZATION (UNDRO). Natural Disasters and Vulnerability Analysis, Report of Experts Group Meeting. Génova, 1979.

URRUTIA, R. y LANZA, C. Catástrofes en Chile 1541-1992. Santiago de Chile: Editorial La Noria, 1993.

VARGAS, G.; FARÍAS, M.; CARRETIER, S.; TASSARA, A.; BAIZE, S. \& MELNICK, D. Coastal uplift and tsunami effects associated to the 2010 Mw8.8 Maule earthquake in Central Chile. Andean Geology, 2011, № 38, p. 219 -238.

VALDOVINOS, C.; MUÑOZ, M.; VÁSQUEZ, D. y OLMOS, V. Desastres naturales y biodiversidad: el caso del humedal costero Tubul-Raqui. Revista Sociedad Hoy, 2012, No 19 , p. 33-52.

VÁSQUEZ, D. Delimitación y Zonificación de los Humedales Costeros de Lenga y TubulRaqui: Orientaciones para la Planificación Territorial de la Región del Bío-Bío. Concepción: Memoria para optar al Título de Geógrafo, Universidad de Concepción, Chile, 2009 (Inédito).

YAMAZAKI, Y.; CHEUNG, K. \& KOWALIK, Z. Depth-integrated, non-hydrostatic model with grid nesting for tsunami generation, propagation and run-up. International Journal for Numerical Methos in Fluids, 2010, DOI:10.1002/fld.2485.

YAMAZAKI, Y. \& CHEUNG, K. Shelf resonance and impact of near-field tsunami generated by the 2010 Chile earthquake. Geophysical Research Letters, 2011, Vol. 38, p. L12605 